\title{
La tabla periódica como fundamento para el aprendizaje de la Química y la construcción de conocimiento
}

\author{
(D) Manuel F. Molina C.*, (D) Liliam A. Palomeque-Forero \\ Grupo de Enseñanza de la Química, Departamento de Química, Facultad de Ciencias, \\ Universidad Nacional de Colombia, Bogotá, D.C., Colombia
}

\begin{abstract}
Resumen
Este escrito sobre la tabla periódica busca ofrecer ideas para la celebración del Año Internacional de la Tabla Periódica propuesto por la UNESCO para este 2019 y apoyado por diferentes organizaciones a nivel internacional. Tales ideas giran alrededor de la consideración de que el lenguaje científico es el eje articular para enseñarla y aspiran a exponer los aportes de diferentes científicos para lograr lo que hoy conocemos como tabla periódica. Además, se hacen algunas propuestas metodológicas para celebrar este aniversario de forma colectiva o personal. (C) 2019. Acad. Colomb. Cienc. Ex. Fis. Nat.
\end{abstract}

Palabras clave: Tabla periódica; Lenguaje; Mendeléyev; Historia; Conocimiento científico.

The periodic table as a foundation for learning chemistry and building knowledge

\begin{abstract}
This paper on the periodic table seeks to offer ideas around the celebration of the International Year of the Periodic Table (2019 IYPT) proposed by UNESCO for 2019 and supported by different international organizations. These ideas revolve around the consideration of scientific language as an articulating axis to teach the periodic table and the use of the contributions of different scientists to achieve what we know today as the periodic table. Additionally, we make some methodological proposals to celebrate the 2019 IYPT collectively or in a personal way. (C) 2019. Acad. Colomb. Cienc. Ex. Fis. Nat.
\end{abstract}

Key words: Periodic table; Language; Mendeléyev; History; Scientific knowledge.

\section{Introducción}

En el contexto actual, en que basta abrir una aplicación en un teléfono para encontrar los datos sobre cada uno de los elementos químicos, los estudiantes no comprenden la importancia de aprender lo relacionado con la tabla periódica en sus clases introductorias de Química tanto en secundaria como en los primeros años de educación superior. Para estas nuevas generaciones, inmersas en la información inmediata y resumida de las herramientas tecnológicas, cada vez son más necesarias las prácticas innovadoras en el aula. Con las nuevas aproximaciones a la enseñanza de la tabla periódica, es posible emplearla como eje conductor del proceso de apropiación del lenguaje de la Química. El manejo adecuado de los nombres, los símbolos, las nomenclaturas y los demás detalles de escritura científica, es uno de los puntos críticos de la "alfabetización química" de los jóvenes. El trabajo con la tabla desde esta perspectiva puede contribuir a la adquisición de elementos del lenguaje químico de manera fluida. También resulta pertinente usarla en el aula para analizar cómo se lleva a cabo la construcción del conocimiento científico. La comprensión de las acciones individuales y colectivas y la revisión de las biografías y las historias personales de los investigadores que han contribuido en diferentes momentos históricos a deducir y organizar la información, favorece el acercamiento de los jóvenes a la Química y les permite reflexionar sobre sus actores y apreciar sus características humanas, sus contradicciones, sus equivocaciones y dificultades, y sus esfuerzos $\mathrm{y}$ triunfos.

\section{El lenguaje de la Química}

Se ha discutido ampliamente el papel que juega el lenguaje en la apropiación de los conceptos científicos. Considerando que el aprendizaje depende y está relacionado con elementos culturales y sociales, el manejo del lenguaje específico es determinante en las etapas generales de acercamiento al conocimiento (memorización, comprensión, elaboración de patrones mediante la interrelación de varios conceptos), así como para extrapolar lo aprendido, con el fin de emplearlo

\footnotetext{
*Correspondencia:

Manuel F. Molina C.; mfmolinac@unal.edu.co

Recibido: 7 de junio de 2019

Aceptado: 26 de junio de 2019

Editor: Sonia Moreno
} 
para resolver situaciones en diversos escenarios y contextos. El aprendizaje de la tabla periódica de los elementos químicos no pasa, en la mayoría de los casos, de la etapa de memorización; los alumnos recuerdan información desconectada de su realidad. La memorización de los nombres de los elementos de la tabla no implica que los hayan relacionado o interpretado, y que le reconozcan alguna utilidad, sentido y objetivo al tema como parte del currículo de ciencias en secundaria e, incluso, a nivel universitario (Galagovsky, et al., 2013). El aprendizaje no puede quedarse, entonces, en la memorización de los términos, por el contrario, debe propiciarse la construcción del lenguaje científico a partir de los propios estudiantes (del Carmen Olivera, et al., 2015); lo anterior implica que se logre un aprendizaje en el que predomine la creatividad y la participación consciente de los implicados (Cervantes \& Gutierrez, 2014).

A pesar de que siempre se han desarrollado opciones lúdicas para incrementar el interés en la información contenida en la tabla y para propiciar el aprendizaje de los detalles más relevantes (Joag, 2014, Martí-Centelles \& Rubio-Magnieto, 2014), a nivel superior puede ser útil abordar el tema con actividades de mayor complejidad, como la redacción de ensayos argumentativos en los que los estudiantes expongan sus ideas y la búsqueda de información sobre la importancia de los elementos químicos, por ejemplo, en el diseño y fabricación de distintos objetos de uso cotidiano (equipos de comunicación, alimentos, materiales cerámicos, circuitos eléctricos, entre muchos otros). La búsqueda de información sobre los nombres de los elementos y sus respectivas propiedades químicas, permite mejorar el manejo del lenguaje técnico oral y escrito, enriquece el vocabulario asociado con la ciencia y, a la larga, genera interés por el origen de los vocablos, los nombres y las denominaciones. De hecho, la escritura sobre estos temas se puede emplear como estrategia de aprendizaje en las aulas
(Rodríguez, 2018) para lograr, no solo la alfabetización científica básica, sino verdaderos avances en la apropiación de las ideas, en su interpretación y su argumentación.

\section{La construcción de conocimiento científico y la tabla periódica}

La celebración de este Año Internacional de la Tabla Periódica (International Year of the Periodic Table, 2019 IYPT) constituye una ocasión inmejorable para enseñar acerca de la construcción del conocimiento científico con base en las ideas que llevaron a concebir lo que hoy conocemos como la tabla periódica. Si bien el conocimiento va más allá de la simple acumulación de datos, los científicos trabajan sobre esa acumulación, la cual no es solo una simple suma de artículos o ideas, y tiene mucho que ver con las tensiones y consensos en torno a un tema (un ejemplo actual es el consenso alrededor de las causas del cambio climático) (Lewandowsky, 2013). La celebración del IYPT nos permite rescatar un área poco tratada en la enseñanza de la Química, la naturaleza de la ciencia y el conocimiento científico, y lograr un espacio para que los profesores se conviertan en alfabetizadores de la ciencia, algo que parece difícil y a veces abstracto.

La tabla periódica constituyó mucho más que una simple clasificación de elementos químicos conocidos desde la antigüedad. Ha sido una herramienta conceptual que predijo nuevos elementos, pronosticó relaciones no reconocidas, sirvió como dispositivo correctivo y cumplió un rol único como dispositivo de memoria y organización. Se ha convertido en una potente carta de síntesis de conocimiento, donde es posible leer, si hacemos la indagación, la historia de la humanidad (Schwartz, 2007). Por ello, un primer ejercicio de construcción partiría de ver cómo han ido apareciendo los diferentes elementos químicos a lo largo del tiempo, utilizando una línea temporal como la de la Figura 1, similar

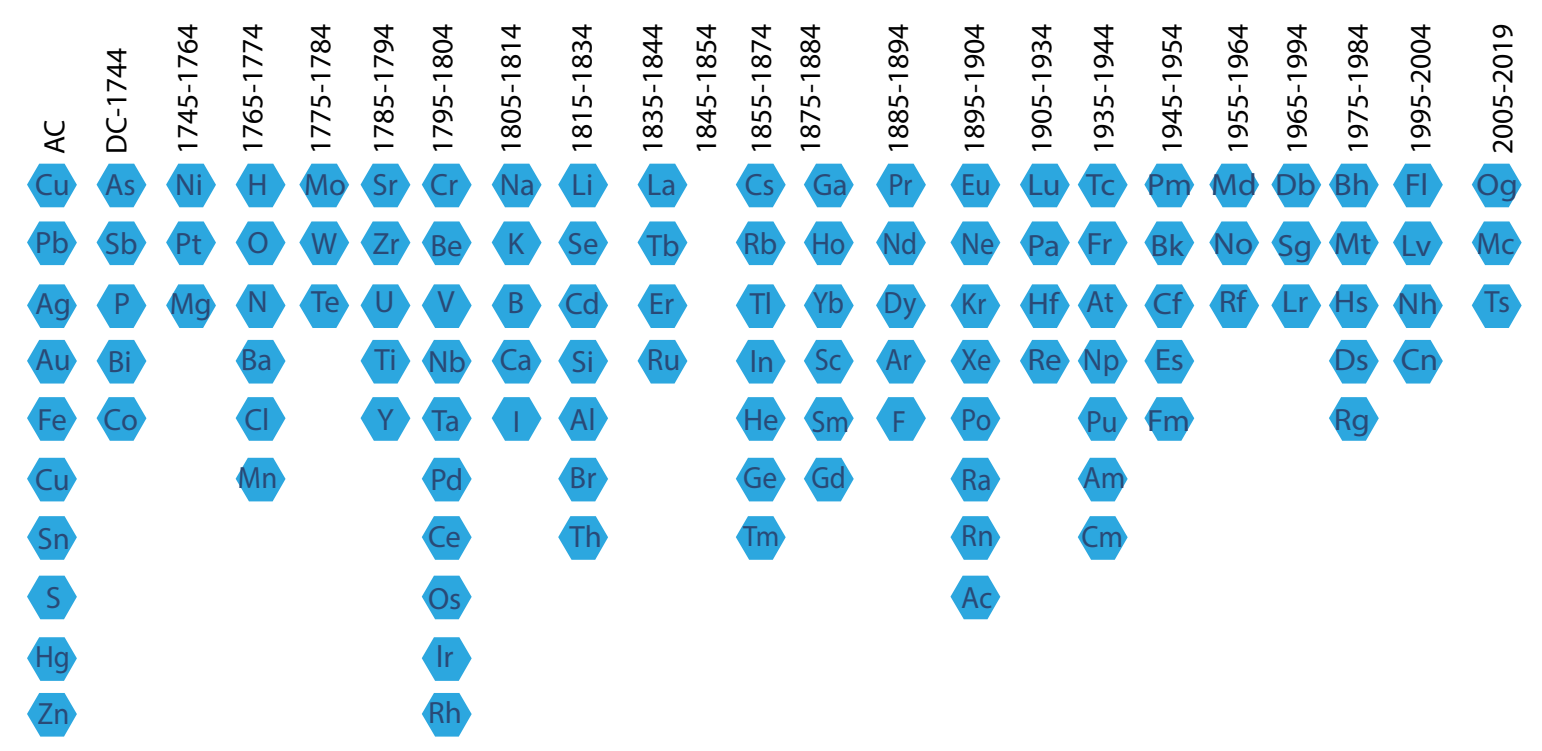

Figura 1. Línea del tiempo de la aparición de los elementos químicos construida en la Waterloo University 
a la propuesta por la Universidad de Waterloo (Waterloo University, 2019), donde apreciamos que, por ejemplo, antes de Cristo ya se contaba con el cobre, el hierro o el oro, elementos muy utilizados en nuestra era moderna. Al recorrer este esquema, podemos relacionar hechos que en cada intervalo de tiempo ayudaron a construir conocimiento.

Por ejemplo, los gases nobles, que se ubican entre 1895 y 1904 (excepto el helio, descubierto en 1868), fueron una prueba de fuego para la tabla periódica de Mendeléyev. El descubrimiento del argón trajo incertidumbre sobre su posición en la tabla, ya que no era un gas diatómico, como el oxígeno, el flúor o el nitrógeno, y tenía un peso atómico de 40 , superior al del potasio, con lo que su ubicación se hacía más difícil. Ello demostró que no bastaba con ordenar los elementos según sus pesos atómicos, que había algo más allá de esta variable. Es el caso del argón, sobre el cual Mendeléyev llegó a sugerir que se trataba de un gas triatómico, el "N $\mathrm{N}_{3}$ " (Scerri, 2001), pero finalmente, se lo ubicó en un grupo nuevo entre los halógenos y los metales alcalinos.

Hasta la publicación de la primera tabla periódica de Mendeléyev (1869) se conocían 63 elementos, los cuales marcaron importantes periodos de la humanidad. Son conocidas las eras del cobre y el hierro, con características especiales cada una. Cuando el cobre era el material más utilizado, las armas tenían cierta capacidad, que se mejoró con las aleaciones de estaño o zinc, pero la situación tuvo un avance revolucionario con las herramientas hechas de hierro y, posteriormente, de acero. Asimismo, bastaría llamar la atención sobre cómo la introducción del aluminio producido en masa significó un cambio en las construcciones o en la fabricación de aviones ( $\sin$ aluminio no podríamos tener aviones rápidos y eficientes), para dimensionar lo que hacen los elementos en nuestra vida. Así pues, la figura 1 puede emplearse desde muchas perspectivas y constituye un material con abundante información implícita que vale la pena introducir en la enseñanza de las ciencias, pues vemos que la ubicación de los elementos en la línea de tiempo lleva consigo una enorme carga de información histórica y conceptual, por lo que constituye una gran puerta de entrada a la descripción de la construcción de conocimiento científico.

Por otra parte, un insumo muy necesario para dar forma a la tabla periódica moderna fue el concepto de elemento. Postulado desde la antigüedad por los griegos en la idea de los cuatro elementos, solo tomó forma con Lavoisier en el siglo XVIII, y se vio nutrido con las contribuciones de científicos como Proust o Berzelius. La introducción de los postulados de Dalton (1803), especialmente el de que un mismo tipo de átomos compone cada elemento, ayudó a construir el concepto de peso atómico relativo, clave en el desarrollo de las ideas de periodicidad. Todo esto, junto con las nociones de molécula de Avogadro (1811), el descubrimiento de nuevos elementos químicos y la determinación más precisa de los pesos atómicos, conformó la sopa de ideas que alcanzó ebullición en el congreso de Karlsruhe en 1860. No puede concebirse la formulación de la ley periódica en las propuestas de la tabla sin tener en cuenta estas ideas y, sobre todo, los pesos atómicos, lo que permite ver que en la construcción del conocimiento se nutre de muchas ideas que confluyen y hacen su aporte.

Una contribución importante, que le dio fulgor especial a la tabla periódica, fueron las triadas de Döbereiner (1817), quien fue el primero en notar la existencia de relaciones en varios grupos de tres elementos con propiedades químicas similares. Así, encontramos la triada del cloro, el bromo y el iodo, en la que el peso atómico del bromo se puede obtener con el promedio del peso del cloro y el iodo $(\mathrm{Cl}=35,470 \mathrm{y}$ $\mathrm{I}=126,470$ ), lo que da 80,970, muy cercano al actual, que es de 79,904. Las triadas revelaron más que relaciones numéricas, mostraron relaciones químicas entre los constituyentes, bien reflejadas en algunos casos, pero en otros, como en la triada de carbono, nitrógeno y oxígeno, no ajustaban. Para 1864 había 63 elementos, pero las triadas no reunían todos los elementos, por lo cual el reto de la periodicidad seguía vigente (Scerri, 2008) Figura 2.

Las triadas parecen ser el camino que siguió Mendeléyev para lograr la aceptación de la ley periódica que propuso, aunque las discusiones de los historiadores se dividen frente a si lo más importante fue la adaptación de los

\begin{tabular}{|c|c|c|c|c|c|c|c|}
\hline \multicolumn{4}{|c|}{ Masa atómica (1850) } & \multicolumn{4}{|c|}{ Número atómico } \\
\hline $\mathbf{L i}$ & 7 & & & Li & 3 & & \\
\hline $\mathrm{Na}$ & 23 & $(7+39) / 2=$ & 23 & $\mathrm{Na}$ & 11 & $(3+19) / 2=$ & 11 \\
\hline K & 39 & & & K & 19 & & \\
\hline & & & & & & & \\
\hline $\mathrm{Ca}$ & 40 & & & $\mathrm{Ca}$ & 20 & & \\
\hline $\mathrm{Sr}$ & 87 & $(40+137) / 2=$ & 88,5 & $\mathrm{Sr}$ & 38 & $(20+56) / 2=$ & 38 \\
\hline $\mathrm{Ba}$ & 137 & & & $\mathrm{Ba}$ & 56 & & \\
\hline & & & & & & & \\
\hline $\mathbf{P}$ & 31 & & & $\mathbf{P}$ & 15 & & \\
\hline As & 75 & $(31+122) / 2=$ & 76,5 & As & 33 & $(15+51) / 2=$ & 33 \\
\hline $\mathrm{Sb}$ & 122 & & & $\mathrm{Sb}$ & 51 & & \\
\hline & & & & & & & \\
\hline$S$ & 32 & & & $\mathrm{~S}$ & 16 & & \\
\hline $\mathrm{Se}$ & 78 & $(32+128) / 2=$ & 80 & $\mathrm{Se}$ & 34 & $(16+52) / 2=$ & 34 \\
\hline $\mathrm{Te}$ & 128 & & & Te & 52 & & \\
\hline & & & & & & & \\
\hline $\mathrm{Cl}$ & 35,5 & & & $\mathrm{Cl}$ & 17 & & \\
\hline $\mathrm{Br}$ & 80 & $(35,5+127) / 2=$ & 81,3 & $\mathrm{Br}$ & 35 & $(17+53) / 2=$ & 35 \\
\hline 1 & 127 & & & 1 & 53 & & \\
\hline
\end{tabular}

\begin{tabular}{|l|l|l|l|l|l|l|l|l|}
\hline $\mathrm{H}$ & \multicolumn{9}{|c|}{} & $\mathrm{He}$ \\
\hline $\mathrm{Li}$ & $\mathrm{Be}$ & & $\mathrm{B}$ & $\mathrm{C}$ & $\mathrm{N}$ & $\mathrm{O}$ & $\mathrm{F}$ & $\mathrm{Ne}$ \\
\hline $\mathrm{Na}$ & $\mathrm{Mg}$ & & $\mathrm{Al}$ & $\mathrm{Si}$ & $\mathrm{P}$ & $\mathrm{S}$ & $\mathrm{Cl}$ & $\mathrm{Ar}$ \\
\hline $\mathrm{K}$ & $\mathrm{Ca}$ & & $\mathrm{Ga}$ & $\mathrm{Ge}$ & $\mathrm{As}$ & $\mathrm{Se}$ & $\mathrm{Br}$ & $\mathrm{Kr}$ \\
\hline $\mathrm{Rb}$ & $\mathrm{Sr}$ & & $\mathrm{In}$ & $\mathrm{Sn}$ & $\mathrm{Sb}$ & $\mathrm{Te}$ & $\mathrm{I}$ & $\mathrm{Xe}$ \\
\hline $\mathrm{Cs}$ & $\mathrm{Ba}$ & & $\mathrm{Tl}$ & $\mathrm{Pb}$ & $\mathrm{Bi}$ & $\mathrm{Po}$ & $\mathrm{At}$ & $\mathrm{Rn}$ \\
\hline
\end{tabular}

Figura 2. Algunas triadas exitosas de Döbereiner y su ubicación en la tabla periódica (elaboradas a partir de Scerri, 2008) 
elementos existentes o las predicciones realizadas (Brito, 2005). Al examinar lo que hizo con las triadas, podemos tomar partido. Mendeléyev hizo una comparación de pesos atómicos de la siguiente forma:

$$
\begin{array}{llll}
23(\mathrm{Na}) & 39(\mathrm{~K}) & 85(\mathrm{Rb}) & 133(\mathrm{Cs}) \\
2 \mathrm{Li} ?(7 \text { o 14) } & 24(\mathrm{Mg}) & 65(\mathrm{Zn}) & 112(\mathrm{Cd})
\end{array}
$$

Aquí surgen dudas sobre si el elemento referido era el litio u otro, lo cual no fue claro en sus comunicaciones (Scerri, 2008); también se observa que sus primeras relaciones fueron horizontales, no verticales, y puede apreciarse el espíritu de las triadas. En sus predicciones la noción de triadas es rescatable, como la que sigue:

\begin{tabular}{|c|c|c|}
\hline & S (32) & \\
\hline As(75) & Se ? & Br (80) \\
\hline & Te (127,5) & \\
\hline
\end{tabular}

donde el peso atómico del selenio se determina con bastante exactitud al relacionar los elementos horizontales y verticales:

$$
(32+75+80+127,9) / 4=79
$$

De esta forma, las triadas, aunque aisladas, fueron árboles primordiales del gran bosque llamado tabla periódica, y se convirtieron en bases del juego que llevó a Mendeléyev y a los demás científicos a proponer relaciones más amplias entre los elementos. A este aporte debe sumarse el de Newlands, a quien se atribuye una hazaña anterior a la de Mendeléyev por sugerir la inversión del telurio y el iodo, dejando a este más a la derecha, lo cual vemos en la tabla periódica actual, aun cuando el telurio posee mayor masa atómica que el iodo. Newlands fue más allá de la anécdota de comparar las octavas musicales con la organización de los elementos, ya que mostró una organización de los elementos conocidos hasta ese momento con las masas atómicas (Figura 3).

Debe recordarse también a Lothar Meyer, quien se disputa con Mendeléyev el honor de ser el "padre" de la tabla periódica, pero cuya tabla, que debió publicarse en 1868, solo fue publicada 25 años después (Figura 4).

Los nombres, los símbolos, las definiciones y los hechos históricos que rodean la aparición de la tabla periódica sirven para alfabetizar en ciencias y para ayudar a comprender cómo se da forma al conocimiento científico mediante el análisis de esos pequeños cambios aportados por cada científico como pilares de una ciencia colectiva, la cual constituye una visión más real de lo que verdaderamente sucede con los avances científicos y desvirtúa la forma predominante de adjudicarlos a una sola persona, opacando, así, las pequeñas contribuciones de los diversos científicos. La tabla periódica, entonces, no puede atribuírsele únicamente a Mendeléyev, ni a Meyer, ya que su perfeccionamiento se debe a la contribución de, por lo menos, cinco científicos (aunque sería posible citar a 15 , o incluso más, pues no cuentan exclusivamente quienes trabajaron directamente en la idea de una tabla periódica, sino también quienes aportaron conceptos relacionados). Una ciencia colectiva desmitifica al héroe

\begin{tabular}{|c|c|c|c|c|c|c|c|}
\hline No. & No. & No. & No. & No. & No. & No. & No. \\
\hline $\mathrm{H} 1$ & F 8 & $\mathrm{Cl} 15$ & $\mathrm{Co} \& \mathrm{Ni} 22$ & $\mathrm{Br} 29$ & $\mathrm{Pd} 36$ & 142 & Pt \& Ir 50 \\
\hline Li2 & $\mathrm{Na} 9$ & K 16 & $\mathrm{Cu} 23$ & $\mathrm{Rb} 30$ & $\operatorname{Ag} 37$ & Cs 44 & Os 51 \\
\hline G 3 & $\mathrm{Mg} 10$ & Ca 17 & Zn 24 & Sr 31 & $\mathrm{Cd} 38$ & $\mathrm{Ba} \& \vee 45$ & $\mathrm{Hg} 52$ \\
\hline Bo 4 & Al 11 & $\mathrm{Cr} 19$ & Y 25 & Ce \& La 33 & U 40 & Ta 46 & Tl 53 \\
\hline C 5 & Si 12 & Ti18 & $\ln 26$ & Zr 32 & Sn 39 & W 47 & $\mathrm{~Pb} 54$ \\
\hline N 6 & $\mathrm{P} 13$ & $\mathrm{Mn} 20$ & As 27 & Di \& Mo 34 & Sb 41 & $\mathrm{Nb} 48$ & Bi 55 \\
\hline 07 & $\mathrm{~S} 14$ & $\mathrm{Fe} 21$ & Se 28 & Ro \& Ru 35 & Tc 43 & $\mathrm{Au} 49$ & Th 56 \\
\hline
\end{tabular}
solitario, desmitifica un trabajo individual y pone de relieve que todos podemos aportar un grano de arena (Tabla 1).

\begin{tabular}{|c|c|c|c|c|c|c|c|}
\hline 1 & 2 & 3 & 4 & 5 & 6 & 7 & 8 \\
\hline & & $\mathrm{Al}=27,3$ & $\mathrm{Al}=27,3$ & & & & $C=12,00$ \\
\hline & & & & & & & 16,5 \\
\hline & & & & & & & $\mathrm{Si}=28.5$ \\
\hline \multirow[t]{5}{*}{$\mathrm{Cr}=52,6$} & $M n=55,1$ & $F e=56,0$ & $C_{0}=58,7$ & $\mathrm{Ni}=58,7$ & $\mathrm{Cu}=63,5$ & $Z n=65,0$ & 44.5 \\
\hline & 49,2 & 48,9 & 47,8 & & 44,4 & 46,9 & 44.5 \\
\hline & $R u=104,3$ & $\mathrm{Rh}=103,4$ & $P d=106,0$ & & $\mathrm{Ag}=107,9$ & $C d=111,9$ & $S n=117,6$ \\
\hline & $92,8=246,4$ & $92,8=246,4$ & $93=2,465$ & & $88,8=244,4$ & 88,3 & $89,4=241,7$ \\
\hline & $\mathrm{Pt}=197,1$ & & $0 s=199$ & & $\mathrm{Hg}=200.2$ & $\mathrm{Hg}=200,2$ & $\mathrm{~Pb}=207,0$ \\
\hline \multirow[t]{3}{*}{9} & 10 & 11 & 12 & 13 & 14 & 15 & 16 \\
\hline & & & $\mathrm{Li}=7,03$ & $\mathrm{Be}=9,03$ & & & \\
\hline & & & 16,02 & 14,7 & & & \\
\hline$N=14,4$ & $0=16,0$ & $F=19,0$ & $\mathrm{Na}=23,05$ & $M g=24,0$ & & & \\
\hline 16,96 & 16,07 & 16,46 & 16,08 & 16 & & & \\
\hline$P=31,0$ & $\mathrm{~S}=32,07$ & $\mathrm{Cl}=35,46$ & $K=39,13$ & $\mathrm{Ca}=40,0$ & $\mathrm{Ti}=48$ & $M_{0}=92,0$ & \\
\hline 44 & 46.7 & 44,5 & 46.3 & 47,6 & 42 & 45 & \\
\hline$A s=75,0$ & $\mathrm{Se}=78,8$ & $\mathrm{Br}=79.9$ & $\mathrm{Rb}=85,4$ & $\mathrm{Sr}=87,6$ & $Z r=90,0$ & $V d=137,0$ & \\
\hline 45,6 & 49.5 & 46,8 & 47,6 & 49,5 & 47,6 & 47 & \\
\hline $\mathrm{Sb}=120,6$ & $T e=128,3$ & $I=126,8$ & $C s=133,0$ & $B a=137,1$ & $T a=137,6$ & $W=184,0$ & \\
\hline $87,4=243,7$ & & & 71 & & & & \\
\hline $\mathrm{Bi}=208.0$ & & & $T e=204.0$ & & & & \\
\hline
\end{tabular}

Figura 3. Organización periódica propuesta por Newlands en 1865 (elaborada a partir de Scerri, 2017)

Figura 4. Tabla periódica propuesta por Meyer en 1868 y publicada en 1895 (elaborada a partir de Scerri, 2017)

Tabla 1. Algunos de los científicos predecesores de Mendeléyev

\begin{tabular}{cccc}
\hline 1829 & 1862 & 1866 & 1868 \\
\hline $\begin{array}{c}\text { Johan } \\
\text { Döbereiner }\end{array}$ & $\begin{array}{c}\text { Alexandre-Emile } \\
\text { Beguyer de } \\
\text { Chancourtois }\end{array}$ & $\begin{array}{c}\text { John Alexander } \\
\text { Newlands }\end{array}$ & $\begin{array}{c}\text { Julius } \\
\text { Lothar } \\
\text { Meyer }\end{array}$ \\
\hline
\end{tabular}




\section{La celebración del año internacional de la tabla periódica}

La celebración de los 150 años de la tabla periódica es un hecho muy significativo para todos los interesados en promover la ciencia. Es una ocasión para participar con entusiasmo y es por ello que queremos hacer las siguientes sugerencias en torno a actividades que podrían desarrollarse.

\section{Para los profesores}

1. Hacer videos sobre los diferentes elementos químicos que sean cortos (3 minutos) y con un contenido que no se limite únicamente a datos sino que incorpore ideas innovadoras. En este sentido, uno de los autores de este ensayo, el profesor Molina, organizó una convocatoria dirigida a docentes de colegio para la cual ya se han recibido alrededor de 200 videos sobre diferentes elementos químicos que pueden verse en el canal de "YouTube Manuel F Molina", en la lista de reproducción "Mi Elemento-Vídeos Tabla Periódica" (https://www.youtube.com/watch? v=PoTlVHr15z s\&list=PL3pSzpYZBdHOhtK7A39_ryHUAwf8T_ VO3\&index $=1$ ).

Construir objetos tridimensionales para representar las propiedades de cada elemento, tales como cubos, triángulos, octaedros, etc.

Con respecto a los cubos, este proyecto se describirá en una próxima entrega sobre la tabla periódica. Serán cubos con información de cada elemento, algunos de tamaño pequeño (10x10) y otros grandes (80x80), cuyo fin es hacer una exposición de los elementos químicos y un concurso de conocimientos. Este proyecto cuenta con el apoyo de la American Chemical Society (ACS). Esta tabla periódica hecha con cubos será emblema del "3-Festival de Química", a realizarse el 23 de octubre de 2019 en las instalaciones del Departamento de Química de la Universidad Nacional de Colombia, sede Bogotá, el cual ya se ha organizado en años anteriores (Molina \& Carriazo, 2019).

2. Elaborar murales para dejar una huella de la tabla periódica en diferentes lugares (salones, laboratorios, paredes, etc.). Los murales pueden ser de la tabla periódica tradicional o propuestas de representación que incluyan otra forma de verla.

3. Concursos sobre el conocimiento de los diferentes elementos. En el sitio de internet de la IUPAC (2019) es posible participar en un desafío de preguntas relacionadas con los elementos químicos (https://iupac.org/100/ pt-challenge/). La siguiente pregunta es un ejemplo de las incluidas en dicha página:

\section{¿Cómo se descubrió el fósforo?}

a) Por un alquimista que intentaba encontrar la piedra filosofal.

b) Su descubrimiento se describe en la Biblia.

c) Accidentalmente, por el famoso químico Berzelius.

d) Por un minero al intentar disolver la roca de fosfato con ácidos.

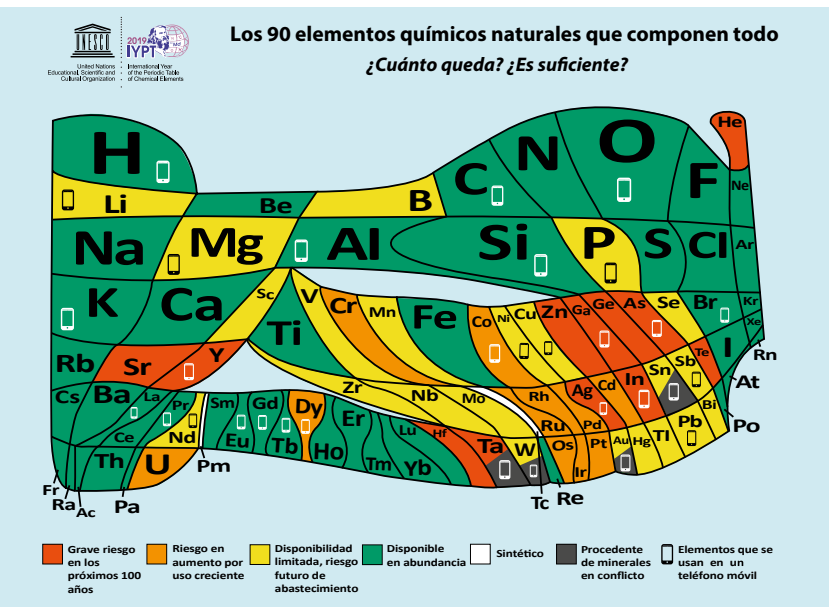

Figura 5. Tabla periódica de la abundancia de los 90 elementos naturales (Tomada de: https://www.euchems.eu/euchems-periodictable/)

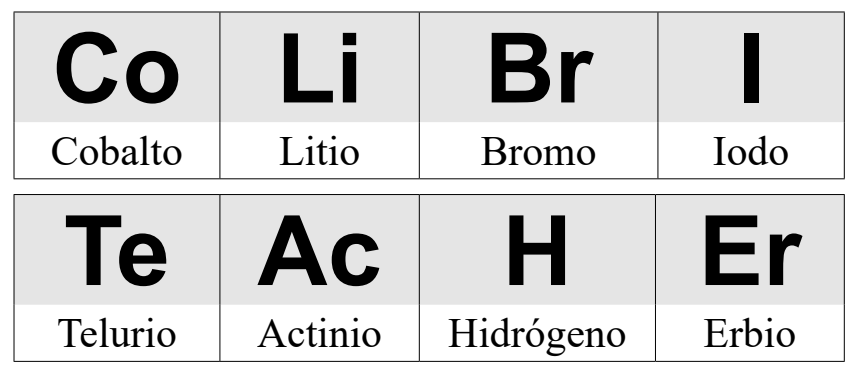

Figura 6. Palabras en español e inglés construidas con símbolos de elementos químicos

4. Hacer presentaciones, conferencias, trabajos y otras actividades relacionadas con los usos y la abundancia de los elementos químicos. Para esto se puede utilizar la tabla periódica de la Figura 5, propuesta por la European Chemical Society (https://www.euchems.eu/), a partir de la cual se pueden tomar uno o varios elementos y estudiar su abundancia a nivel global, sus usos, las cuestiones sociales que se suscitan en torno a su obtención, su reciclaje y posibles soluciones para disminuir el consumo de aquellos elementos que poseen un grave riesgo de disponibilidad.

\section{Para personas interesadas}

1. Imprimir una camiseta, una gorra, una bufanda u otra prenda con el elemento favorito o una alusión a la tabla periódica (Figura 6).

2. Regalar una tabla periódica como obsequio de fechas especiales o de recordatorio de viajes. Existen diferentes representaciones de la tabla disponibles en papelerías o en tiendas virtuales.

3. Aprender acerca de los elementos químicos. Hay páginas de internet que ofrecen información e imágenes impactantes de los diferentes elementos químicos, por ejemplo, https://periodictable.com/ 


\section{Conclusiones}

La celebración del Año Internacional de la Tabla Periódica haciendo énfasis en la enseñanza del lenguaje de la Química y la historia de la ciencia contribuye a formar personas que entiendan el proceso de la construcción de conocimiento y a divulgar la Química de forma adecuada. Unámonos a esta importante celebración y exaltemos su ícono: 2019 IYPT.

\section{Contribución de los autores}

El presente escrito surgió de un acuerdo de los autores, que contribuyeron por igual a su elaboración y redacción. LPF contribuyó especialmente con la parte del lenguaje de la Química y MMC con lo concerniente a la construcción de conocimiento.

\section{Conflicto de intereses}

Ninguno que declarar.

\section{Referencias}

Brito, A., Rodríguez, M. A., Niaz, M. (2005). A reconstruction of development of the periodic table based on history and philosophy of science and its implications for general chemistry textbooks. Journal of Research in Science Teaching: The Official Journal of the National Association for Research in Science Teaching. 42 (1): 84-111.

Cervantes, E. \& Gutiérrez, P. R. (2014). Actitudes de los estudiantes de bachillerato ante la educación científica. Congreso Iberoamericano de Ciencia, Tecnología, Innovación y Educación. Universidad Autónoma de Ciudad Juárez, Juárez (México).

del Carmen Olivera, A., Mazzitelli, C. A., Guirado, A. M. (2015). El conocimiento construido por los alumnos en las clases de Química. Revista Electrónica de Enseñanza de las Ciencias. 14 (1): 77-94.

European Chemical Society. (2019). EuChemS Periodic Table. Fecha de consulta: 21 de junio de 2019. Disponible en: https://www.euchems.eu/

Galagovsky, L. R., Rodríguez, M. A., Stamati, N., Morales, L. F. (2003). Representaciones mentales, lenguajes y códigos en la enseñanza de ciencias naturales. Un ejemplo para el aprendizaje de concepto de "reacción química" a partir del concepto de "mezcla". Enseñanza de las ciencias: revista de investigación y experiencias didácticas. 21 (1): 107-121.
IUPAC. (2019). Periodic Table Challenge. Fecha de consulta: 21 de junio de 2019. Disponible en: https://iupac.org/100/ptchallenge/

Joag, S.D. (2014). An effective method of introducing the periodic table as a crossword puzzle at the high school level. Journal of Chemical Education. 91 (6): 864-867.

Lewandowsky, S., Gignac, G. E., Vaughan, S. (2013). The pivotal role of perceived scientific consensus in acceptance of science. Nature Climate Change. 3 (4): 399-402.

Martí-Centelles, Vicente; Rubio-Magnieto, J. (2014). ChemMend: A Card Game To Introduce and Explore the Periodic Table while Engaging Students' Interest. Journal of Chemical Education. 91 (6): 868-871.

Molina, M. F. (2019). Mi Elemento-Vídeos Tabla Periódica. Fecha de consulta: 21 de junio de 2019. Disponible en: https:// www.youtube.com/watch?v=PoTlVHr15zs\&list=PL3pSzp YZBdHOhtK7A39_ryHUAwf8T_VO3\&index=1)

Molina, M. F. \& Carriazo, J. G. (2019). Awakening Interest in Science and Improving Attitudes toward Chemistry by Hosting an ACS Chemistry FeSTiVAl in Bogotá, Colombia. Journal of Chemical Education. 96 (5): 944-950

PeriodicTable. (2019). The Photographic Periodic Table of the Elements. Fecha de consulta: 21 de junio de 2019. Disponible en: https://periodictable.com

Rodríguez, B. F. C. \& Hernández, T. E. (2016). El lenguaje como una estrategia para el aprendizaje de los temas del currículo de las ciencias. Bio-grafía. Escritos sobre la biología y su enseñanza. 9 (17): 73-88.

Scerri, E. (2008). The role of triads in the evolution of the periodic table: Past and present. Journal of Chemical Education. 85 (4): 585.

Scerri, E. \& Worrall, J. (2001). Prediction and the Periodic Table. Studies in History and Philosophy of Science. 32A: 407-452.

Schwartz, A. T. (2007). The Periodic Table: Its Story and Its Significance (Eric R. Scerri). New York, Estados Unidos de América: Oxford University Press.

Scerri, Eric. (2017). El descubrimiento de la tabla periódica como un caso de descubrimiento simultáneo. Epistemología e Historia de la Ciencia. 2 (1): 82-97.

Waterloo University. (2019). iypt-2019-timeline-elements-poster. Fecha de consulta: 21 de junio de 2019. Disponible en: https://uwaterloo.ca/chem13-news-magazine/may-2019/ feature/iypt-2019-timeline-elements-poster 